4. Mahieu-Caputo D, Dommergues M, Delezoide AL, et al. Twin to twin transfusion syndrome. Role of the fetal renin-angiotensin system. Am J Pathol2000; $156: 629-36$.

5. Barr M, Cohen MM. ACE inhibitor fetopathy and hypocalvaria. The kidney-skull connection. Teratology $1991 ; 44: 485-95$
6. Martinovic J, Benachi A, Laurent N, et al. Fetal toxic effects of angiotensin II receptor antagonists. Report of three additional cases. Lancet $2001 ; 358: 241-2$.

7. Schutz S, Le Moullec JM, Corvol P, Gasc JM. Early expression of all components of the reninangiotensin-system in human development. Am J Pathol 1996; 149 : 2067-79.
8. Gubler MC, Sarrut S, Imbert MC, et al. Renal tubular dysgenesis, an autosomal recessive disorder and the renin-angiotensin system. J Am Soc Nephrol1993 ; $4: 263$.

9. Gribouval 0, Gonzales M, Neuhaus T, et al. Mutations in genes in the renin-angiotensin system are associated with autosomal recessive renal tubular dysgenesis. Nat Genet 2005 ; 37 : 964-8.

\title{
NOUVELLE
}

\section{Nociception : un grand rôle pour les petits protons}

Philippe Séguéla
Institut Neurologique de Montréal,

Département de neurologie et neurochirurgie,

Université McGill,

3801, rue University, Montréal (Québec), H3A 2B4 Canada. philippe.seguela@mcgill.ca
> Un nombre croissant de preuves expérimentales indiquent que les protons $\left(\mathrm{H}^{+}\right)$, les plus simples entités matérielles, sont aussi des neurotransmetteurs. Parmi ces preuves figure en bonne place la découverte récente d'une famille de canaux ioniques neuronaux directement activés par les protons, appelés ASIC (acid sensing ion channels), dans le système nerveux central et périphérique [1]. Par leurs propriétés d'activation par les $\mathrm{pH}$ acides, ces récepteurs-canaux excitateurs pourraient favoriser le développement des douleurs chroniques induites par les acidoses tissulaires; de plus, leur activité pourrait aggraver les dommages tissulaires associés aux accidents ischémiques [2, 3].

La perception de la douleur provient initialement de l'excitation d'une population de neurones périphériques qui forment les fibres nerveuses $C$ et/ou $A \delta$ multimodales. Ces nocicepteurs spécialisés dans la détection des stimulus dangereux pour l'intégrité tissulaire sont situés principalement dans les ganglions spinaux et dans les ganglions trijumeaux. Les électrophysiologistes savent depuis longtemps que les neurones centraux et périphériques peuvent répondre à une application de solutions acides par des courants cationiques rapides [4]. Ces courants dépolarisants rapides offrent une grande perméabilité aux ions $\mathrm{Na}^{+}$ et sont sensibles à l'amiloride, deux caractéristiques du rôle des ASIC. Les sous-unités ASIC sont des protéines intégrales à 2 domaines transmembranaires

qui appartiennent à la classe génétique des canaux sodiques épithéliaux $\mathrm{ENaC}$ et des dégénérines de nématodes [5]. La

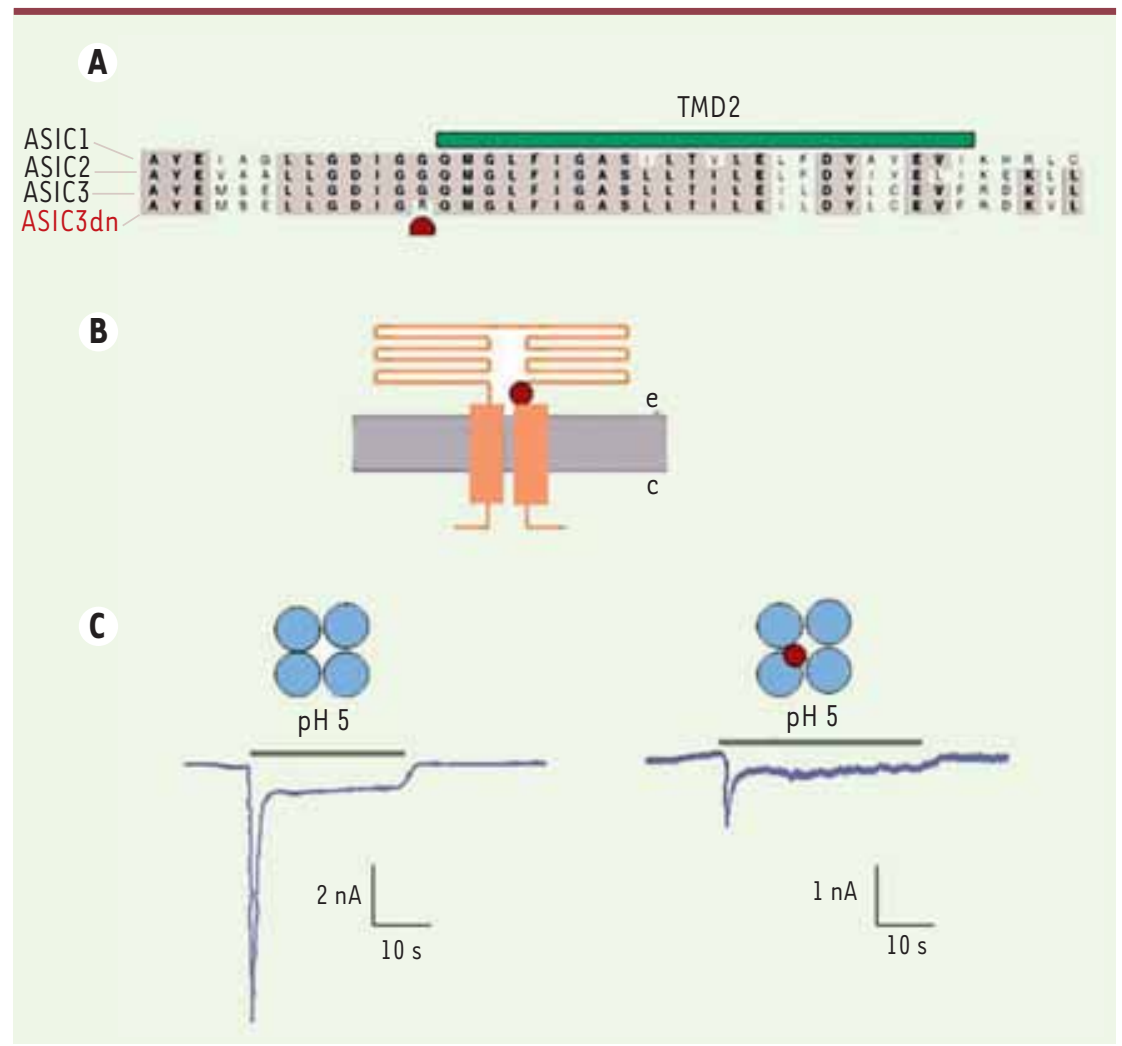

Figure 1. Effet dominant négatif de la sous-unité ASIC3 mutante G439R. A. La mutation G439R est située dans la partie extracellulaire de la sous-unité ASIC3 proximale au $2^{\mathrm{e}}$ domaine transmembranaire, dans une région critique très conservée au sein de la famille ASIC. $B$. Schéma topologique d'une sous-unité ASIC montrant la localisation de la mutation G439R (e : espace extracellulaire, c: cytoplasme). C. Inhibition fonctionnelle des courants ASIC par intégration de la sous-unité ASIC3 G439R. Notez la différence d'amplitude entre les courants biphasiques médiés par les récepteurs-canaux ASIC3 sauvages et par les récepteurs-canaux hétéromériques formés de sous-unités ASIC3 sauvages et mutantes. 
famille des canaux tétramériques ASIC comprend 4 gènes chez les mammifères: ASIC1-4. ASIC1, 2 et 3 répondent aux protons avec des sensibilités et des cinétiques distinctes; ASIC4 demeure une sous-unité toujours orpheline à ce jour. La sous-unité ASIC3 a la particularité intéressante d'être exclusivement exprimée dans les neurones sensoriels primaires chez les rongeurs et de produire un canal homomérique à cinétique d'activation biphasique constituée d'une phase rapide suivie d'une composante lente et soutenue $[6,7]$. La phase rapide est plus sensible aux protons avec un $\mathrm{pH}_{50}=6,5$, donc compatible avec les conditions physiopathologiques observées dans les cas d'ischémie tissulaire. À ce propos, les fibres sensorielles qui innervent le cœur expriment de hauts niveaux de la sous-unité ASIC3 et sont particulièrement sensibles à l'acidose ischémique, ce qui laisse présager qu'ASIC3 contribue aux douleurs cardiaques comme celles de l'angine de poitrine [8]. D'après les expériences d'ablation génétique chez la souris, les ASIC peuvent former des hétéromères contenant ASICl, ASIC2 et ASIC3 dans les neurones des ganglions spinaux [9]. Nous avons exploité cette capacité d'hétéromérisation entre sous-unités ASIC pour concevoir et développer des souris transgéniques qui expriment la sousunité ASIC3 mutante à effet dominant négatif (ASIC3dn) dans leurs neurones périphériques et centraux [10]. La mutation G439R, que nous avons découverte par criblage fonctionnel, est située dans un motif extracellulaire très conservé dans la famille des canaux ASIC et rend le canal insensible aux stimulations par les protons (Figure 1). Après avoir vérifié qu'ASIC3dn inhibe bien les courants induits par ASICl, 2 et 3 sauvages en systèmes d'expression hétérologue, nous avons «construit» une lignée de souris surexprimant ASIC3dn sous contrôle du promoteur neuronal du neurofilament à chaîne légère. Nous avons observé que les neurones des ganglions spinaux de ces souris transgéniques présentent un déficit marqué dans leurs réponses rapides aux $\mathrm{pH}$ acides, sans être affectés dans leurs réponses lentes connues pour être médiées par les récepteurs-canaux TRPVl également activés par la capsaïcine. Donc la sous-unité ASIC3dn joue bien son rôle de suppresseur sélectif des courants ASIC nés dans les neurones sensoriels. Quelle est la conséquence de cette suppression fonctionnelle dans les réponses des animaux à différents tests de nociception? Nous avons soumis les souris transgéniques homozygotes à des tests thermiques sans pouvoir détecter la moindre différence avec les animaux témoins; ce résultat n'est pas une surprise puisque les canaux ASIC ne jouent aucun rôle dans la thermotransduction. En revanche, les animaux transgéniques montrent une hypersensibilité aux stimulus mécaniques, avec ou sans inflammation induite par injection de formaline ou de zymosan, et après injection intramusculaire de solution saline hypotonique (modèle de Sluka). Ils sont aussi plus sensibles aux stimulus chimiques nociceptifs déclenchés par injection sous-cutanée de formaline ou par injection abdominale d'acide acétique. Ces données suggèrent que, malgré leurs propriétés électrophysiologiques dépolarisantes, les ASIC remplissent une fonction régulatrice importante dans la conversion des signaux nociceptifs en réponse douloureuse, et ce pour des modalités sensorielles bien définies.

$\varepsilon n$ résumé, la diversité des récepteurs ASIC et leur haut niveau d'expression dans certaines populations neuronales démontrent sans ambiguïté l'importance des protons comme premiers messagers, tant au niveau central qu'au niveau périphérique où ils annoncent des stress cellulaires. Nos résultats avec des souris transgéniques hypersensibles qui expriment une sous-unité ASIC3 mutante montrent que les récepteurs-canaux ASIC, qui se manifestent à la surface des neurones sensoriels, sont requis pour une perception adéquate des stimulus douloureux inflammatoires et mécaniques. La composition hétéromérique des ASIC sensoriels chez les rongeurs et chez I'humain n'est pas encore connue mais il ne fait aucun doute que les petits protons et leurs récepteurs ont encore un grand rôle à jouer dans notre compréhension des mécanismes moléculaires de la nociception. $\diamond$

Nociception:

a great role for the small protons

\section{RÉFÉRENCES}

1. Waldmann R, Champigny G, Bassilana F, et al. A proton-gated cation channel involved in acidsensing. Nature 1997 ; 386 : 173-7.

2. Immke DC, McCleskey EW. Lactate enhances the acidsensing $\mathrm{Na}^{+}$channel on ischemia-sensing neurons. Nat Neurosci $2001 ; 4: 869-70$.

3. Xiong ZG, Zhu XM, Chu XP, et al. Neuroprotection in ischemia : blocking calcium-permeable acid-sensing ion channels. Cell $2004 ; 118: 687-98$.

4. Akaike N, Ueno S. Proton-induced current in neuronal cells. Prog Neurobiol $1994 ; 43$ : 73-83.

5. Kellenberger $S$, Schild L. Epithelial sodium channel/ degenerin family of ion channels: a variety of functions for a shared structure. Physiol Rev 2002 ; $82: 735-67$.

6. De Weille JR, Bassilana F, Lazdunski M, et al. Identification, functional expression and chromosomal localisation of a sustained human proton-gated cation channel. FEBS Lett 1998 ; 433 : 257-60.

7. Babinski K, Le KT, Séguéla P. Molecular cloning and regional distribution of a human proton receptor subunit with biphasic functional properties. J Neurochem $1999 ; 72$ : 51-7.

8. Sutherland SP, Benson CJ, Adelman JP, et al. Acidsensing ion channel 3 matches the acid-gated current in cardiac ischemia-sensing neurons. Proc Natl Acad Sci USA $2001 ; 98: 711-6$.

9. Benson CJ, Xie J, Wemmie JA, et al. Heteromultimers of $D \varepsilon G / \varepsilon N a C$ subunits form $\mathrm{H}^{+}$-gated channels in mouse sensory neurons. Proc Natl Acad Sci USA 2002 ; $99: 2338-43$.

10. Mogil JS, Breese NM, Witty MF, et al. Transgenic expression of a dominant-negative ASIC3 subunit leads to increased sensitivity to mechanical and inflammatory stimuli. J Neurosci 2005 ; 25 : 9893-901. 